\title{
An Integrative Review of Scientific Evidence for Reconnective Healing
}

\author{
Ann L. Baldwin, $\mathrm{PhD}^{1,2}$ and Natalie L. Trent, $\mathrm{PhD}^{3}$
}

\section{Abstract}

Background: Biofield therapies offer a novel, non-invasive approach to treating chronic diseases based on assessing and adjusting an individual's physiological and emotional responses through their bio-energetic field. Reconnective Healing ${ }^{\mathrm{TM}}(\mathrm{RH})$ is defined as: "...not just energy healing, but instead a more comprehensive spectrum of healing composed of energy, light, and information."

Objectives: Several biofield therapies, such as Reiki, Therapeutic Touch and Johrei, have already been reviewed in the literature but RH has received little attention even though it is taught and practiced worldwide. This review provides a critical assessment of RH as a healing modality.

Methods: Scientific research articles published in peer-reviewed journals addressing RH were identified using relevant databases and archives. Information was extracted from each article that met selection criteria for evaluation of quality of reporting and design. This review summarizes and critically evaluates the five currently published peer-reviewed research papers involving $\mathrm{RH}$ and assesses whether $\mathrm{RH}$ provides consistent physiological outcomes between the studies.

Results: These results, taken together, suggest: (i) exposure of a healer or healee to RH, either directly or indirectly, amplifies their degree of autonomic arousal and energy, (ii) RH can reduce pain and improve range of motion in people with shoulder limitations, and (iii) when individuals experience RH as a group, their autonomic nervous systems simultaneously show sudden similar responses consistent with the idea that RH is mitigated by entrainment of biofields.

Conclusions: Since these studies are extremely varied in design it is not possible at this point to reach conclusions about the general effectiveness of RH. More clinical and physiological research performed on different populations under a range of conditions is needed in order to support this healthcare approach.

Keywords: Reconnective Healing, biofield therapy, complementary and alternative medicine

\section{Introduction}

The problem of treating chronic diseases

$\mathbf{O}$ UR CURRENT HEALTHCARE system is based largely on Allopathic medicine, in which diagnosis is relied upon to determine the optimal treatment and the area of the body to treat, and drugs are used to treat symptoms of disease. The allopathic system, which is so successful for treating acute illnesses, is not as effective with chronic diseases because drugs taken on a long-term basis can become ineffective or produce unacceptable side-effects, ${ }^{1}$ leading to poor medication adherence. ${ }^{2}$ For example, according to a recent study, approaches to managing chronic lower back pain using opioids have not been very effective $^{3}$ In this study, researchers reviewed the results of 20 randomized controlled trials with a total of 7925 participants. Thirteen of the trials (3419 participants) addressed short-term

\footnotetext{
${ }^{1}$ Department of Physiology, College of Medicine, University of Arizona, Tucson, AZ.

${ }^{2}$ Laboratory for the Advances in Consciousness and Health, Department of Psychology, University of Arizona, Tucson, AZ.

${ }^{3}$ Department of Medicine, Brigham and Women's Hospital Harvard Medical School, Boston, MA.
}

(c) Ann L. Baldwin and Natalie L. Trent, 2017; Published by Mary Ann Liebert, Inc. This article is available under the Creative Commons License CC-BY-NC (http://creativecommons.org/licenses/by-nc/4.0). This license permits non-commercial use, distribution and reproduction in any medium, provided the original work is properly cited. Permission only needs to be obtained for commercial use and can be done via RightsLink. 
effects on chronic low back pain. At least half of participants in these 13 trials withdrew because the drugs did not work or they experienced adverse health events. Overall, the studies showed that opioids may moderately benefit chronic low back pain patients on a short-term basis, but at doses much higher than recommended. There is very little information documenting the efficacy of opioids for back pain on a chronic basis.

Studies further document that older adults with chronic pain cite concerns about adverse drug effects and the use of too many medications as reasons for preferring nonpharmacologic therapies. ${ }^{4}$ As a result, chronic sufferers from such conditions as arthritis, back pain, diabetes, hypertension, heart disease, and cancer may seek out alternative treatments. Individuals with back pain are some of the most frequent users of complementary and alternative medicine (CAM) practices. ${ }^{5}$

On a wider scale, surveys indicate that those with the most serious and debilitating medical conditions, such as cancer, ${ }^{6,7}$ chronic pain, ${ }^{8,9}$ and $\mathrm{HIV},{ }^{10}$ tend to be frequent users of CAM. For this reason, it would be more beneficial for the patients if their primary care physicians were open to both allopathic and CAM types of treatments. However, this kind of integration depends largely on the establishment of an evidence base for safety and effectiveness of CAM approaches. The purpose of this review is to critically assess the scientific evidence for the effectiveness of a worldwide CAM modality, Reconnective Healing $^{\mathrm{TM}}(\mathrm{RH})$, which is classified as a biofield therapy.

\section{Biofield therapy}

Biofield therapies are based on the philosophy that humans have an energetic dimension. Such therapies are believed to balance out disturbance in the energy field caused by physical and psychologic symptoms. Indigenous systems of healing such as Ayurvedic and Chinese medicine, which go back thousands of years, rest on concepts of a vital force or subtle life energy that is central to healing. These subtle energies may actually refer to something similar to the present-day concept of the biofield. Biofield therapies are among the most controversial CAM modalities; they involve the practitioner interacting with the body's bioenergetic field, rather than with the physical body. Measurements of extracellular ion fluxes and voltage potential differences in living organisms led to the hypothesis that an electrostatic field surrounds and penetrates the human body. ${ }^{11,12}$ Endogenous biofields, as detected from the heart (ECG), brain (EEG), and muscle (EMG), are recognized clinically as indices of health and disease. Modern superconducting quantum interference devices have become important tools in clinical medicine for measuring the biomagnetic fields of the heart and brain. ${ }^{13}$

\section{Effects of biofield therapies in clinical populations}

Several therapeutic procedures, based on the use of the hands to sense and alter endogenous biofields, have been developed with the goal of improving physical and psychologic health. Among the most commonly practiced of these biofield therapies are Reiki, Therapeutic Touch, Healing Touch, and external qigong. ${ }^{14} \mathrm{~A}$ recent review concludes that the clinical effectiveness of biofield therapies is strongest in symptom management for pain and cancer and evidence is promising for clinical populations with arthritis, dementia, and heart disease. ${ }^{15}$ A review of biofield therapies not involving physical touch reported that two thirds of the higher scoring trials demonstrated statistically significant beneficial treatment outcomes. ${ }^{16}$ Specifically, Therapeutic Touch reduced pain and/or anxiety in the elderly, in people recovering from surgery, in burn victims, in adults with tension headaches, and in HIV-positive children. In single studies, quality of life was improved by external qigong in cocaine-dependent adults and by Healing Touch in patients with cancer. Reiki reduced psychologic depression in adults. In another review, Anderson and Taylor ${ }^{17}$ described studies showing that Therapeutic Touch improved function in people suffering from arthritis and reduced complications from bone marrow transplant. Healing Touch also significantly decreased the length of hospital stay and anxiety levels in patients who had undergone coronary artery bypass graft, compared to those receiving standard care and visitation. Thrane and $\mathrm{Cohen}^{18}$ reviewed the effect of Reiki for pain and anxiety in randomized clinical trials. Of the 12 papers on the subject, only 7 met the selection criteria (4 with cancer patients, 1 with postsurgical patients, and 2 with older adults). Most of the seven studies yielded statistically significant results either for pain or anxiety or both. Thus, although the scientific evidence is sparse, and sample sizes are mostly small, biofield therapies do appear to be useful for reducing pain and anxiety in various patient populations.

\section{What is Reconnective Healing?}

A therapy that is relatively recent compared to Reiki and qigong and involves interactions with biofields is RH. RH, as discovered and developed by Eric Pearl DC, in the early 1990s, "is not just energy healing, but instead a more comprehensive spectrum of healing composed of energy, light and information. It is not something we do. It's something we allow, become, catalyze and help facilitate." RH is taught worldwide by means of seminars and workshops. Descriptions of the different levels of training are available at thereconnection.com. Similar to some other biofield therapies, in RH, the biofields of both healer and healee are thought to resonate with an external environmental field.

There is evidence that naturally occurring fields can alter biofields and can even change cellular function. For example, Harold Saxton Burr, PhD, of Yale Medical School, showed that changes in environmental electromagnetic fields, such as caused by thunderstorms, substantially affected the biofields of trees. ${ }^{19}$ In another case, biologically generated dynamic frequency information (ECG recordings from a person focusing intentionally on feelings of appreciation) significantly increased DNA synthesis of cultured fibroblasts. ${ }^{20}$ In addition to producing classical electromagnetic fields, oscillating electric dipoles in the body can act as antennae to produce nonclassical fields ${ }^{21}$ that do not fade with distance. ${ }^{22}$ Cells, tissues, and organs each produce certain collective frequencies and it has been hypothesized that environmental fields may alter these frequencies by entraining them so that they resonate with the external field. ${ }^{23}$

\section{What happens during an $\mathrm{RH}$ session?}

During RH training programs, students are trained to concentrate on their clients' energy fields by focusing on the sensations in their own hands. These sensations are not limited to RH; other biofield therapies, such as Reiki, produce sensations such as warmth and tingling in practitioners' hands ${ }^{24}$ that are accompanied by measured increases in microvascular perfusion in the fingers. ${ }^{25}$ During $\mathrm{RH}$, the sensations become 
stronger as practitioners move their hands away from the client's body. The further away they move their hands, the stronger the sensations feel, rather like stretching a rubber band. While the healer is feeling the changes in the sensations in their hands, they are also observing the physical responses of the clients that have a tendency to increase as the practitioners move their hands further and further away from the recipient. These effects are visibly clear and distinct, such as eyelids fluttering uncontrollably, eyes rapidly darting from side to side, and fingers and/or feet twitching. These types of responses have also been reported with other biofield therapies such as Reiki. ${ }^{26}$ Sometimes clients have no recognizable experience at all, or their experience may emerge hours or days after the session. More often, the experiences tend to be immediate and the healing results almost instantaneous. Reconnective Healers soon learn to recognize, through visual observation, a correlation between what they are doing and feeling, and what is happening with their client on the treatment table, although there is no independent evidence for cause and effect.

\section{Rationale for a review of $R H$}

Although people who have given and/or received RH have experienced its beneficial effects first hand, those who are not familiar with RH may be more skeptical. According to Eric Pearl DC, Founder of The Reconnection LLC (Personal Communication), over 20,000 mainstream healthcare practitioners, including MDs, DOs, DCs, PTs, and nurses, have trained in $\mathrm{RH}$ and have incorporated it into their private practices across five continents. This number is based on the fact that The Reconnection LLC records show they have trained over 100,000 people and that $20 \%-30 \%$ are professional healthcare providers. Therefore, it is important to determine whether there are consistent effects on clinical and physiologic outcomes. No efforts have been made to organize, integrate, and synthesize published findings or to prioritize the directions of future research. As stated by Jensen and Onyskiw, ${ }^{27}$ through integrating, organizing, and synthesizing findings from studies of the same phenomena into a rational and coherent pattern, knowledge develops within a discipline. A synthesis of the results of RH research can help develop disciplinary knowledge and provide practitioners with the information they need to advance theory, interventions, patient care, and public policy. For this reason, the peer-reviewed scientific research on $\mathrm{RH}$ is presented here as an integrative review.

\section{Data collection}

Scientific research papers published in peer-reviewed journals addressing $\mathrm{RH}$ were identified using the following databases searched through July 2016: PubMed, CINAHL, PsycINFO, AMED, and Web of Science. The search string employed was "Reconnective Healing”' OR "Energy Healers", OR "Biofield." Supplemental searches were conducted on reference lists from identified papers and on the archives of "Subtle Energies and Energy Medicine."

\section{Selection criteria}

Studies included in this review were (1) English-language, full papers published in peer-reviewed journals; (2) designed to investigate the physiologic effects of RH on human subjects, whether healthy or unhealthy, or on RH practitioners; (3) designed to investigate the effects of RH on groups of people; (4) either controlled or noncontrolled trials; and (4) case studies of patients receiving $\mathrm{RH}$. Studies were excluded that lacked measurement of the effects of RH on human subjects.

\section{Data extraction}

Information was extracted from each paper that met selection criteria for evaluation of quality of reporting and design, and to facilitate demographic categorization. Data

Table 1. Quality Assessment Criteria

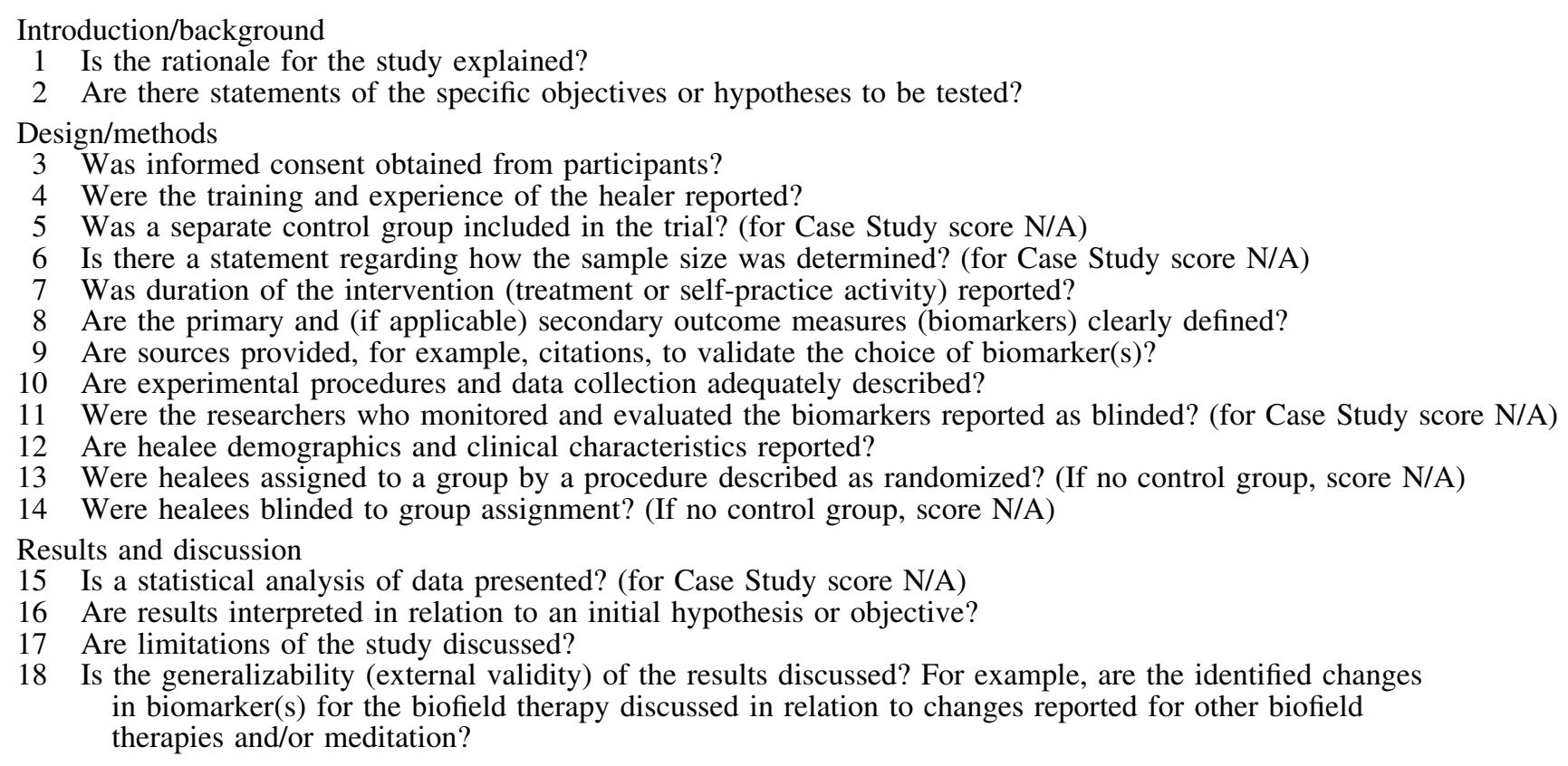

Each criterion is scored as Yes (1), Partial (0.5), No (0), or Not Applicable (N/A). The final percentage score was derived by dividing the total sum of points by the number of items scored (18-no. of N/A items) and multiplying by 100 . 


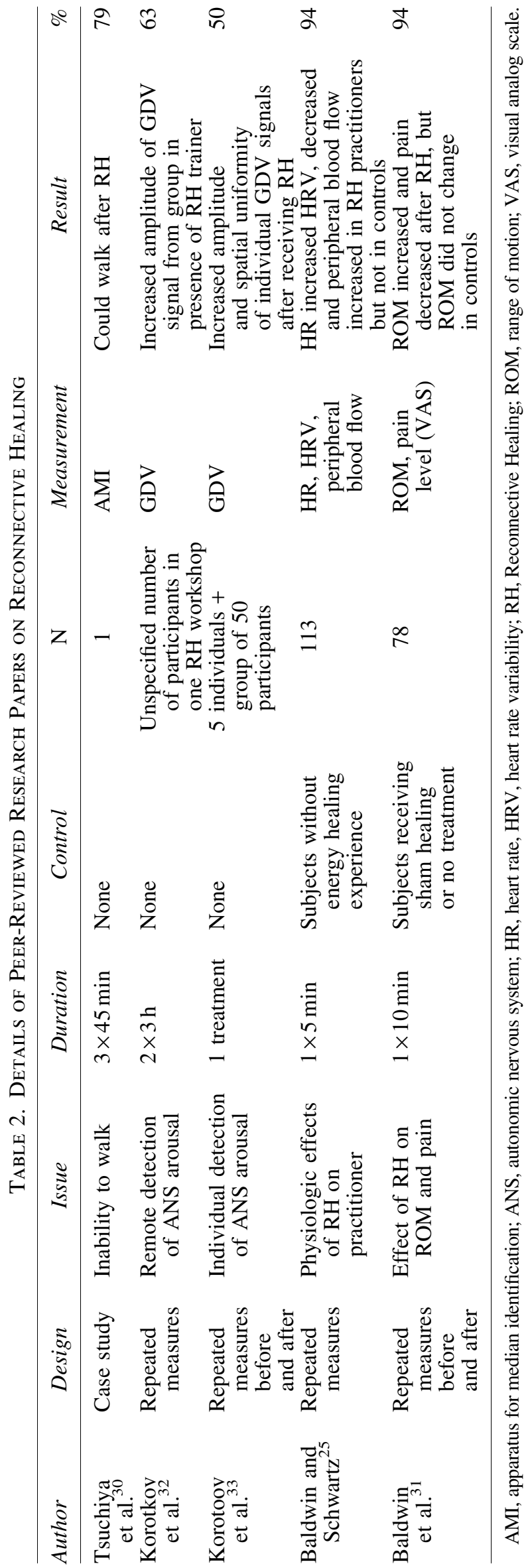

extracted included author(s) and year, experimental design, issue addressed, duration of therapy, whether there was a control group, number of participants receiving treatment, type of measurements and results.

\section{Quality assessment}

Criteria applied to evaluate papers (Table 1) were based, in part, on assessment items developed for CONSORT (Consolidated Standards of Reporting Trials), ${ }^{28}$ and for the CONSORT extension for nonpharmacologic trials. ${ }^{29}$ In developing criteria, the fact that most of the trials that would be evaluated were pilot studies was considered. As such, criteria were worded appropriately, for example, rather than requiring a sample size calculation, it was sufficient only that a statement be included as to how the sample size was decided (item 5). As another example, since early-phase research usually aims to generate rather than test a hypothesis, only a statement of either objectives or hypotheses was necessary (item 2). The criteria were scored independently by both the authors for each of the studies. A Yes (1.0)/Partial $(0.5) / \mathrm{No}(0) / \mathrm{NA}$ scoring system for each item was used to minimize subjectivity. The scores and methodological details of the research papers are listed in Table 2.

\section{Results}

\section{Characteristics of included studies}

Investigation of the various databases using the selected search string provided the following numbers of citations: PubMed (89), CINAHL (69), PsycINFO (59), AMED (27), and Web of Science (174). However, only a total of three papers satisfied the inclusion criteria. All other citations were excluded because they lacked measurement of the effects of RH on human subjects. Two further papers fulfilling inclusion criteria were identified in the archives of "Subtle Energies and Energy Medicine." The five selected papers included a case report, a study of ambient changes during an $\mathrm{RH}$ workshop, a noncontrolled pilot study of healthy volunteers, an RCT of subjects with shoulder limitations, and a study of biomarkers in $\mathrm{RH}$ practitioners during self-healing (Table 2).

Data were reported from a total of 197 individuals, plus a class of an unspecified number of participants across the five included studies. Two studies involved participants with health issues, ${ }^{30,31}$ and three studies involved participants without any stated health problems, ${ }^{25,32,33}$ one of which focused on the RH practitioners themselves. ${ }^{25}$ Only two of the five studies incorporated independent control groups. ${ }^{25,31}$

\section{Scoring of trials}

The five studies were scored for quality of reporting and design with a set of 18 criteria (Table 1); average scores are included in Table 2. Agreement between the scorers was quite high with identical scores for the three highest scoring studies and differences of $9 \%$ and $12 \%$ for the other two studies. Studies that scored less than $60 \%$ were considered "poor," those that scored 60\%-69\% "fair," and those that scored over 69\% "good." Mean percent score was 76 \pm 19 (standard deviation). The criterion that scored lowest was \#18 (generalizability of results). The paper by Korotkov et al. ${ }^{32}$ was scored as a case study because it involved only 
one RH session given to a group of participants. For this paper, item \#12 (are healee demographics and clinical characteristics reported?) was scored NA because the group of participants was just attending an RH seminar. For the paper by Baldwin and Schwartz, ${ }^{25}$ items \#13 (were healees assigned to a group by a procedure described as randomized?) and \#14 (were healees blinded to group assignment?) were scored as NA because the three groups were Reconnective Healers, Reiki masters, and a control group with no energy healing experience; thus, there was no preexperimental group assignment as such.

\section{Biomarkers}

Among the biomarkers of physiologic changes in healees and healers, the most frequently assessed were electrodermal activity $\left(\mathrm{EDA}^{30,32,33}\right)$ and heart rate/heart rate variability (HR/HRV). ${ }^{25,31}$ Other measures, assessed in single studies, were peripheral blood flow, ${ }^{25}$ range of motion $(\mathrm{ROM}){ }^{31}$ and self-reported pain levels. ${ }^{31}$

\section{Electrodermal activity}

All external and internal stimuli, including emotions, are processed by the autonomic nervous system (ANS) leading to alterations in capillary blood flow, production of sweat, and transfer of electrons within the connective tissue. These changes are reflected in the electrical parameters of a person's skin. If a person or body part (usually finger) is placed in a pulsed electromagnetic field produced between two electrodes, some of these electrons are extracted from the skin and subsequently from deeper connective tissues. The free electrons accelerate toward the anode, gaining enough energy to cause further ionization to form an electron avalanche on the surface of the electrode. The electronic "glow" of this discharge can be captured by an optical CCD camera system and translated to a digital computer file. This is the basis of the gas discharge visualizer that measures EDA. ${ }^{34}$ The lack of glow from the gas discharge visualization (GDV) camera is an indicator of the impeded transfer of electron density to the body's tissues, and a diminished flow of free radical reactions. In other words, this is an indicator of a reduction in the energy supply of organs and systems.

Three of the five studies included in this review used EDA as a biomarker. ${ }^{30,32,33}$ The quality of these investigations was mixed, two being of good quality ${ }^{30,32}$ and one of poor quality. ${ }^{33}$ However, all three studies reported an increase in EDA of participants who experienced $\mathrm{RH}$, indicating an enhanced level of physiologic arousal. Only the case study ${ }^{30}$ investigated the effects of RH on an unhealthy individual; the other two experiments ${ }^{32,33}$ involved healthy participants.

The case study subject ( 74 years old) had emaciated limbs and had been unable to walk without a walker for 6 months before the study. This subject received three 45-min healing sessions by a level III Reconnective Healer, one-on-one, while lying on a massage table. EDA measurements were made on the subject's fingers and toes before and after each of the sessions using an apparatus for median identification (AMI) device that measures the electrical conductivity, capacitance, and polarization of skin tissue and fluids. ${ }^{35}$ Further information regarding the correlation of these measures to physiologic changes is described in other studies. ${ }^{36-38}$ After healing, significant changes were observed in the electroconductivity of the body, possibly caused by changes in microvascular blood flow and by sweating. After the third session, the EDA of the lower body was significantly larger (by $60 \%$ ) than that of the upper body, indicating that the sympathetic nervous activity in this area had become substantially more active. Comparing before and after the total healing period, EDA of the lower body increased by $49 \%$ and EDA of the upper body decreased by $22 \%$. This response is consistent with a redistribution of blood flow from the upper to the lower body. Immediately after the third healing session, the subject stood up without help and walked without a walker.

A problem with this study is that the AMI procedure is not well validated. Variable contact area, pressure, skin hydration, and, most importantly, the presence of sweat ducts can all confound AMI devices to one degree or another; therefore, more rigorous clinical studies are needed to assess their validity. ${ }^{39}$ In addition, possible effects of the AMI procedure on the subject, independent of the RH, are not addressed. Further objective neurologic data taken before and after the healing would have strengthened the study.

The second study demonstrated enhancement of physiologic arousal in five healthy volunteers (as shown by increased amplitude and spatial uniformity of stimulated electrophotonic emissions from subjects) after they received $\mathrm{RH}^{33}$ These experiments were performed as part of a workshop held at the 20th annual ISSSEEM (International Society for the Study of Subtle Energy Medicine) Conference, June 2010. More than 50 people attended the workshop. First, a teaching assistant of Eric Pearl DC introduced the concept of RH to all participants. Next, 10 fingertip images were obtained from 5 participants, before and after receiving $\mathrm{RH}$ from the teaching assistant using the GDV electrophotonic camera. Increased EDA was recorded for all five participants (statistically significant for three of them).

The increased physiologic arousal levels of the subjects after they received $\mathrm{RH}$ are consistent with the results of the case study. ${ }^{30}$ However, the lack of standardization of AMI measurements, as mentioned previously, weakens the study. This report was scored as poor quality due to lack of hypothesis or stated objectives, lack of official participant consent, lack of control group, sparsity of experimental details, such as duration of the healing, and no discussion of limitations of the study.

The third study using EDA as a biomarker addressed remote detection of human physiologic arousal during an RH workshop training using a modification of the electrophotonic imaging GDV camera system. ${ }^{32}$ In this study, Korotkov attended an RH training workshop (September 12/ 13, 2008 in Los Angeles, USA), led by Eric Pearl DC, and one of his trained teaching assistants, and set up a GDV device in the room where the training took place. To obtain readings in the room air in general, rather than from the air adjacent to an individual, he had modified the instrument by attaching an antenna to the titanium calibration cylinder designed to create a nonhomogeneous electromagnetic field in space. A generator produced voltage impulses that were applied to the GDV electrode resulting in production of a bias current in the antenna that was dependent on the capacitance of the environmental air. It was assumed that any increases in the signal would reflect enhanced conductivity of skin and underlying tissue of participants in the room 
triggered by increased physiologic arousal. Changes in the electrical conductivity of the air within the room were monitored the night before the workshop and then as the training progressed the next day. There was very little variation in the signal at night when the room was empty.

On both days the signal increased when a speaker/trainer stepped on stage and presented to the audience, compared to recess periods. This response was interpreted as a heightened physiologic arousal of the audience when in the presence of the trainers. The signal also showed increased oscillations during these periods, possibly reflecting an entrainment, or synchronization, of the participants' state of physiologic arousal, all rising and falling in a periodic manner. Thus, all three studies, utilizing EDA as a biomarker in three very different circumstances, demonstrated increased physiologic arousal in the subjects experiencing RH either directly or indirectly.

However, in all three studies, measurement devices were used that have yet not been adequately tested regarding the veracity of the measurements. Although Korotkov has reported some standardization of techniques to show the stability and reliability of the GDV parameters, ${ }^{40,41}$ and the GDV camera has been certified as a medical device by the Committee on New Medical Technique of the Russian Ministry of Health, no standardized basis for interpreting findings has been established. A review of 136 exploratory studies on the application of the GDV as a diagnostic instrument in medicine shows that it gives comparable results to other more standard forms of measurement under a wide variety of conditions. ${ }^{42}$ However, a documented database and device standardization need to be established and published in peer-reviewed journals to validate the GDV camera as a truly scientific instrument.

Assuming that the GDV measurements did accurately reflect increases in the level of physiologic arousal and connectedness of the audience as they experienced the presence of Reconnective Healers and witnessed the healings, these results suggest that the RH workshop activities influenced the emotions of audience participants. In partial support of this assumption, correlations have been reported between the GDV signal and various parameters of HRV, an established tool for assessing autonomic function, in healthy people. ${ }^{43}$ However, the audience could have been reacting to charismatic speakers, with results having nothing to do with RH. To solve this dichotomy, it would be necessary to repeat the experiment in the presence of a non-RH motivational speaker.

Another problem is that no mechanism was postulated by which changes in the autonomic physiologic arousal of people throughout the room could affect the electrical conductance of room air in the area of the GDV. As Korotkov states in this publication: "To prove or disapprove these ideas we need to have a series of further experiments with different modalities of healing." In addition, further experiments are required to compare the data obtained from the $\mathrm{RH}$ workshop with the effects of groups of people at church services, sports rallies, political gatherings, and other highly emotional events.

\section{$H R$ and HRV}

Two of the five studies included in this review used HR and HRV as biomarkers, one in RH practitioners them- selves $^{25}$ and the other in subjects with impaired ROM who received $\mathrm{RH}$ as a therapy, one-on-one with the healer. ${ }^{31}$ These two studies were scored as good quality because they fulfilled most of the criteria listed in Table 1 . HRV is a noninvasive measure of the complementary relationship between the sympathetic and parasympathetic branches of the $\mathrm{ANS}^{44}$ and reflects the capacity of the central autonomic network, including the prefrontal cortex, central nucleus of the amygdala, hypothalamus, and brainstem, to respond and adapt to environmental demands. ${ }^{45} \mathrm{HRV}$ is also correlated with the ability to regulate one's emotions. ${ }^{46} \mathrm{HRV}$ usually increases with enhanced vagal stimulation, such as produced by yoga, and a type of guided meditation in which respiration rate is reduced, ${ }^{47,48}$ and decreases with sympathetic arousal as produced in times of stress or focused, applied effort. $^{49}$

When 31 advanced Reconnective Healers practiced on themselves, HR did not change; however, HRV significantly decreased compared to baseline. In contrast, Reiki masters and control subjects showed no significant changes in HRV. ${ }^{25}$ The sudden decrease in HRV seen when the Reconnective Healers began self-healing is consistent with decreased parasympathetic nervous activity and application of mental effort. It would be interesting to determine whether or not this state of mental focus, as monitored by decreased HRV and/or some other more specific biomarker of increased sympathetic activity, is correlated with an increased effectiveness of healing by Reconnective Healers.

This HRV response was not observed in the 17 subjects who received $\mathrm{RH}$ for their impaired $\mathrm{ROM}^{31}$ and their HRV did not change, although their HR decreased after the healing similar to control subjects who just relaxed on the massage table.

\section{Other biomarkers}

Peripheral blood flow. In the experiments on selfpractice by Reconnective Healers, ${ }^{25}$ cutaneous blood perfusion (amount of blood flow flowing through a defined region of interest) of the fingers was also measured. This measurement was made because of anecdotal evidence that Reiki practitioners often feel their hands heat up when they practice. Measurements were made on 50 experienced Reconnective Healers and 31 Reiki masters 5 min before, 5 min during, and 5 min after a self-healing. Corresponding measurements were made on 32 control subjects, who gazed at a calming picture in place of self-healing. Changes in blood perfusion were assessed by asking subjects to place their hand, palm down, on a mat and then scanning the middle three fingers using a laser Doppler perfusion imager. Comparing finger perfusion at the start point with the endpoint of RH self-healing, perfusion increased by $10.3 \%$ more than for control subjects $(p=0.003)$. Reiki practitioners showed a greater increase in perfusion $(17.5 \%$ more than control subjects, $p=0.001)$. Both RH and Reiki groups showed similar perfusion at baseline. The increase in finger blood perfusion shown, on average, by Reconnective Healers and Reiki masters, was probably caused by local release of vasodilators into the blood. It is not clear from this experiment whether an increase in finger blood perfusion is a marker of a more efficient healer; it may simply indicate that the practitioner has entered into a healing state. This 
response requires further investigation to determine what it may signify.

\section{ROM and self-reported pain}

Although RH has been reported by practitioners to alleviate many common clinical conditions, such as rheumatoid arthritis, tendonitis, neck pain, pain from fibromyalgia, allergies, and eczema, until recently there has been little published scientific evidence to support these claims. In this placebo-controlled, randomized, clinical trial, RH (one 10min treatment) was assessed to determine whether it could significantly increase ROM and reduce pain in subjects with a variety of shoulder limitations. Participating subjects ( $n=78,41: 37 \mathrm{~F}: \mathrm{M}$ ) were randomly assigned to one of five experimental groups: RH, Reiki, Sham Healing, Physical Therapy, or rest. The number of subjects chosen was based on a power analysis at $80 \%$ power.

Before their treatment, all participants were asked to stand with their back to a wall, without touching it, with their arms at their sides. They were then video recorded as they moved their arms out to the sides and then up toward their head, in a scapular plane as far as they could go, while keeping their arms straight. The videos were later analyzed to determine the angle each arm made above the horizontal when the participants reached their full ROM. Participants were asked to rate their maximum pain as they completed the movement on a scale from 1 to 10 . Next they entered the treatment room and received their treatment or rest, while lying supine on a massage table. Apart from those in the rest group, participants were not told what type of treatment they were receiving. After the treatment or rest, the ROM recordings and pain assessments were repeated.

RH significantly increased participants' ROM by over $26^{\circ}$ on average compared to Reiki and to Physical Therapy that gave improvements of $20^{\circ}$ and $12^{\circ}$, respectively, compared to $3^{\circ}$ for the rest-only group. The effect size was 0.8 , which is considered a large effect size. These results cannot be explained by a placebo effect because sham treatment did not significantly improve ROM. Pain scores decreased on average by $24 \%$ after $\mathrm{RH}$ compared to $10.1 \%$ after Reiki and $11 \%$ after Physical Therapy. This pilot study suggests that the use of RH is at least as effective as Physical Therapy in improving ROM in patients with painful shoulder limitation when evaluated immediately after a 10-min treatment.

One limitation of this study was that the participants who were assigned to the rest group knew they were in that group, and this introduces a certain amount of bias.

Another limitation is that inferences drawn from the results should be confined to those seen in a single 10-min treatment session with no follow-up. Further studies to evaluate such issues as the time-course of the effect of Physical Therapy, Reiki, and RH and the outcome on disability and function are warranted. There is a clear clinical need for nonsurgical treatments that are safe and effective for chronic, painful shoulder problems.

\section{Analysis and Conclusions}

The present review identified five studies; a case study, ${ }^{30}$ effects of $\mathrm{RH}$ charismatic leaders addressing conference attendees on ambient energy level, ${ }^{32} \mathrm{RH}$ treatments on five randomly selected volunteers, ${ }^{33}$ physiologic changes in $\mathrm{RH}$ healers after performing $\mathrm{RH}^{25}$ and a well-designed RCT. ${ }^{31}$ Since these studies are extremely varied in design, it is not possible at this point to reach firm conclusions about the general effectiveness of RH.

In order for RH to be accepted in the medical field, it is essential to establish an evidence base for its safety and effectiveness and to demonstrate that it has consistent effects on clinical and physiologic patient outcomes. Although the one clinical case study demonstrated that after receiving $\mathrm{RH}$, an individual with an incapacitated lower half of the body was able to walk, there was no control of possible confounding factors. On the contrary, the recently published randomized, placebo-controlled shoulder study provides robust clinical data from 78 individuals with limited ROM, demonstrating that a short session of RH is at least as effective as an equal duration of Physical Therapy in improving ROM and pain relief. Longer term follow-up studies are needed to test whether the benefits of $\mathrm{RH}$ are sustained.

Like other biofield therapies, further studies are needed to determine whether RH consistently alleviates particular disorders. Such trials would be stronger if they included both conventional and biofield-related endpoints rather than basing conclusions on measurements such as GDV and AMI; such measures have not been adequately validated and tested. The use of robust research methods, including the use of blinding and control treatments as well as large enough sample sizes for statistical validity, is essential in all future experiments to test the effectiveness of $\mathrm{RH}$. Innovative areas for future study of RH include clinically useful RH protocols that can be implemented in fastpaced clinical environments and investigation of the basic mechanistic principles responsible for observed health benefits.

Regarding clues as to the possible mechanisms for mediation of $\mathrm{RH}$, one common finding from the three published nonclinical RH studies is that exposure of healer or healee to RH, either directly or indirectly, amplifies their autonomic physiologic arousal and increases their energy as shown by GDV and HRV. In the two GDV studies performed in a group context, the autonomic responses of individuals within each group to $\mathrm{RH}$ appeared to be synchronized, consistent with the claim that Reconnective Healers can access environmental fields that influence their own biofields, and that these changes are passed onto their patients' biofields by entrainment. More specific recommendations for how to better test the above claim would be to:

(1) Simultaneously measure the galvanic skin response (GSR) and HRV of pairs of RH healers and healees before and during the healing process.

(2) Simultaneously measure GSR and HRV of individuals in a group with and without the presence of a nonRH motivational speaker or a Reconnective Healer who is engaged in the healing process and is also monitored.

Such experiments would determine whether the previously observed changes in the biofields of Reconnective Healers were passed onto their patients by entrainment and 
would contribute to a better understanding of the $\mathrm{RH}$ process.

\section{Author Disclosure Statement}

This paper and the studies by Baldwin and Schwartz ${ }^{25}$ and Baldwin et al., ${ }^{31}$ were partially funded by The Reconnection; this funding source did not play any part in the analysis of data nor in the writing of the papers.

\section{References}

1. Rao JK, Mihaliak K, Kroenke K, et al. Use of complementary therapies for arthritis among patients of rheumatologists. Ann Intern Med 1999;131:409-416.

2. Garner JB. Problems of nonadherence in cardiology and proposals to improve outcomes. Am J Cardiol 2010;105: 1495-1501.

3. Shaheed CA, Maher CG, Williams KA, et al. Efficacy, tolerability and dose-dependent effects of opioid analgesics for low back pain. A systematic review and meta-analysis. J Am Med Assoc Intern Med 2016;176:958-968.

4. Park J, Lavin R, Couturier B. Choice of nonpharmacological pain therapies by ethnically diverse older adults. Pain Manag 2014;4:389-406.

5. Murthy V, Sibbritt DW, Adams J. An integrative review of complementary and alternative medicine use for back pain: A focus on prevalence, reasons for use, influential factors, self-perceived effectiveness, and communication. Spine J 2015;15:1870-1883.

6. Sikorskii A, Wyatt GK, Siddiqi A, et al. Recruitment and early retention of women with advanced breast cancer in a complementary and alternative medicine trial. Evid Based Complement Alternat Med 2011;2011:734517.

7. Mao JJ, Farrar JT, Xie SX, et al. Use of complementary and alternative medicine and prayer among a national sample of cancer survivors compared to other populations without cancer. Complement Ther Med 2007;15:21-29.

8. Tan $\mathrm{MG}^{1}$, Win MT, Khan SA. The use of complementary and alternative medicine in chronic pain patients in Singapore: A single-centre study. Ann Acad Med Singapore 2013;42:133-139.

9. Denneson LM, Corson K, Dobscha SK. Complementary and alternative medicine use among veterans with chronic noncancer pain. J Rehabil Res Dev 2011;48:1119-1128.

10. Littlewood RA, Vanable PA. The relationship between CAM use and adherence to antiretroviral therapies among persons living with HIV. Health Psychol 2014;33:660-667.

11. Burr HS, Northrop FSC. Evidence for the existence of an electro-dynamic field in living organisms. Proc Nat Acad Sci 1935;25:284-288.

12. Becker RO, Selden G. The Body Electric: Electromagnetism and the Foundation of Life. New York, NY: William Morrow, 1985.

13. Kleiner R, Koelle D, Ludwig F, Clarke J. Superconducting quantum interference devices: State of the art and applications. Proc IEEE 2004;92:1534-1548.

14. Levin J. Energy healers: Who they are and what they do. Explore 2011;7:13-26.

15. Jain S, Hammerschlag R, Mills $P$, et al. Clinical studies of biofield therapies: Summary, methodological challenges, and recommendations. Global Adv Health Med 2015; 4(suppl):58-66.
16. Hammerschlag R, Marx BL, Aiken M. Non-touch biofield therapy: A systematic review of human randomized controlled trials reporting use of only nonphysical contact treatment. J Atern Complement Med 2014;20:881-892.

17. Anderson JG, Taylor AG. Biofield therapies in cardiovascular disease management: A brief review. Holist Nurs Pract 2011;25:199-204.

18. Thrane S, Cohen SM. Effect of Reiki therapy on pain and anxiety in adults: An in-depth literature review of randomized trials with effect size calculations. Pain Manag Nurs 2014;15:897-908.

19. Burr HS. Blueprint for Immortality. London, UK: Neville Spearman, 1971.

20. Rein G. The in vitro effect of bioenergy on the conformational states of human DNA in aqueous solutions. Acupuncture Electrother Res 1995;20:173-180.

21. Frolich $\mathrm{H}$. Long range coherence and energy storage in biological systems. Int J Quantum Chem 1968;2:641-649.

22. Tesla N. Transmission of energy without wires. Sci Am (Suppl) 1904;57:237-260.

23. Rein G. Bioinformation within the biofield. Beyond Boelectromagnetics. J Altern Comp Med 2004;10:59-68.

24. Singg S. Use of Reiki as a biofield therapy: An adjunct to conventional medical care. Clin Case Rep Rev 2015;1: 54-60.

25. Baldwin AL, Schwartz GE. Physiological changes in energy healers during self-practice. Complement Ther Med 2012;20:299-305.

26. Complementary \& Alternative Therapies in Nursing. 6th edition. In: Snyder M, Lindquist R, eds., Part 3. Energy and Biofield Therapies. New York, NY: Springer Publishing Company, 2010:276.

27. Jensen LA, Onyskiw JE. Making sense of bits of information (editorial). Western J Nurs Res 2003;25:117-118.

28. Schulz KF, Altman DG, Moher D. CONSORT Group. CONSORT 2010 statement: Updated guidelines for reporting parallel group randomized trials. Ann Intern Med 2010;152:726-732.

29. Boutron I, Moher D, Altman DG, et al. CONSORT Group. Extending the CONSORT statement to randomized trials of nonpharmacologic treatment: Explanation and elaboration. Ann Intern Med 2008;148:295-309.

30. Tsuchiya K, Harada T, Motoyama H. Study of body's energy changes in non-touch energy healing 2 . Reconnective Healing performed on a subject suffering from emaciated lower limbs. Subtle Energies Energy Med 2010;21:29-40.

31. Baldwin AL, Fullmer K, Schwartz GE. Comparison of physical therapy with energy healing for improving range of motion in subjects with restricted shoulder mobility. Evid Based Complement Alternat Med 2013, 9.

32. Korotkov K, Orlov D, Madappa K. New approach for remote detection of human emotions. Subtle Energies Energy Med 2008;19:111-126.

33. Korotkov K, DeVito D, Arem K, et al. Healing experiments assessed with electrophotonic camera. Subtle Energies Energy Med 2010;20:1-8.

34. Nagayama N, Motoyama H. Electrical properties in the extremities along meridians in patients with unilateral pulmonary tuberculosis. Subtle Energies Energy Med 2007;18:9-19.

35. Motoyama H, Smith WT, Harada T. Pre-polarization resistance of the skin as determined by the single square voltage pulse method. Psychophysiology 1984;21:541-550. 
36. Motoyama H. Measurements of Ki Energy Diagnoses \& Treatments. Shukyo Shinri Shuppan, Tokyo: Human Science Press, 1997.

37. Chevalier G, Mori K. The effect of earthing on human physiology Part 2: Electrodermal measurements. Subtle Energies Energy Med 2007;18:11-34.

38. Ahn AC, Martinsen OG. Electrical characterization of acupuncture points: Technical issues and challenges. $\mathrm{J}$ Altern Complement Med 2007;13:817-824.

39. Kostyuk N, Cole P, Meghanathan N, et al. Gas discharge visualization: An imaging and modeling tool for medical biometrics. Int J Biomed Imaging 2011;3:1-7.

40. Korotkov K. Aura and Consciousness. St Petersburg, Russia: Russian Ministry of Culture, State Editing and Publishing Unit, 1999.

41. Korotkov KG, Popechitelev EP. Method for gas-discharge visualization and automation of the system of realizing it in clinical practice. Med Tekh 2002;1:21-25.

42. Korotkov KG, Matravers P, Orlov DV, Williams BO. Application of electrophoton capture analysis based on gas discharge visualization technique in medicine: A systematic review. J Altern Complement Med 2010;16: 13-25.

43. Cioca GH, Giacomoni P, Rein G. A correlation between GDV and heart rate variability measures: A new measure of well being. In: Konstantin G, Korotkov, eds. Measuring Energy Fields: Current Research. Fair Lawn, USA: Backbone Publishing Co, 2004:59-64.
44. Malik M, Heart rate variability: Standards of measurement, physiological interpretation, and clinical use. Circulation 1996;93:1043-1065.

45. Thayer JF, Friedman BH. Stop that! Inhibition, sensitization, and their neurovisceral concomitants. Scand J Psychol 2002;43:123-130.

46. Geisler FC, Vennewald N, Kubiak T, Weber H. The impact of heart rate variability on subjective well-being is mediated by emotion regulation. Person Indiv Diff 2010;49:723-728.

47. Melville GW, Chang D, Colagiuri B, et al. Fifteen minutes of chair-based yoga postures on guided meditation performed in the office can elicit a relaxation response. Evid Based Complement Altern Med 2012;2012:10.1155/501986.

48. Tay K, Baldwin AL. Effects of breathing practice in vinyasa yoga on heart rate variability in university students: A pilot study. Yoga Phys Ther 2015;5:214

49. Uusitalo A, Mets T, Martinmaki K, et al. Heart rate variability related to effort at work. Appl Ergon 2011;42:830-838.

Address correspondence to:

Ann L. Baldwin, PhD

Department of Physiology

Arizona Health Sciences Center

Rm. 4103, 1501 N. Campbell

P.O. Box 245051

Tucson, AZ 85724

E-mail: abaldwin@u.arizona.edu 